Article

\title{
Real-World Use and Outcomes of ALK-Positive Crizotinib-Treated Metastatic NSCLC in US Community Oncology Practices: A Retrospective Observational Study
}

\author{
Craig Reynolds ${ }^{1,2}$, Elizabeth T. Masters ${ }^{3}$, Jenny Black-Shinn ${ }^{2}$, Marley Boyd ${ }^{2}$, Jack Mardekian ${ }^{3}$, \\ Janet L. Espirito ${ }^{2, *}$ and Marc Chioda ${ }^{3}$ \\ 1 Florida Cancer Specialists and Research Institute, Ocala, FL 34471, USA; chrmd@hotmail.com \\ 2 The US Oncology Network/McKesson Specialty Health, The Woodlands, TX 77380, USA; \\ jblackshinn15@gmail.com (J.B.-S.); marley.boyd@mckesson.com (M.B.) \\ 3 Pfizer Oncology, New York, NY 10017, USA; elizabeth.masters@pfizer.com (E.T.M.); \\ jack.mardekian@pfizer.com (J.M.); marcd.chioda@pfizer.com (M.C.) \\ * Correspondence: janet.espirito@mckesson.com; Tel.: +1-832-566-6779
}

Received: 8 May 2018; Accepted: 15 May 2018; Published: 29 May 2018

\begin{abstract}
Introduction: Around 3-5\% of non-small cell lung cancers (NSCLC) are ALK-positive. Crizotinib was the first approved ALK inhibitor from clinical trials. However, there are less data on the utilization and patient outcomes associated with crizotinib in real-world clinical practice. Methods: This was a retrospective, observational study of adult crizotinib-treated ALK-positive metastatic NSCLC patients who received treatment between 1 September 2011 and 31 October 2014, with follow up through 31 December 2015. Data were obtained via programmatic queries of the US Oncology Network/McKesson Specialty Health electronic health record database, supplemented with chart abstraction. Overall survival (OS) and time to treatment failure (TTF) were estimated from crizotinib initiation using the Kaplan-Meier (KM) method. Results: Of the $n=199$ ALK-positive crizotinib-treated patients meeting eligibility criteria, crizotinib was prescribed as first line $(1 \mathrm{~L})$ in $n=123(61.8 \%)$. The majority (88.9\%) had confirmed adenocarcinoma histology and $32.2 \%$ had brain metastases at initial diagnosis. Median age at crizotinib initiation was 60.2 years (range 27.1-88.2); $54.8 \%$ were never smokers, $33.7 \%$ were former smokers. Treatment of $250 \mathrm{mg}$, twice daily, was most commonly prescribed $(89.5 \%)$ with the dose unchanged from an initial dose in $79.4 \%$ of patients. The primary discontinuation reason was progression $(n=91,58.7 \%)$. Patients $(3.2 \%)$ were identified as discontinuing crizotinib as a result of treatment-related toxicity. With median follow-up time of 13.0 months ( $\min -\max =0.03-46.6$ ), median OS from crizotinib initiation was 33.8 months (95\% CI $=24.3-38.8)$. Median TTF was 10.4 months. Conclusions: Crizotinib usage evaluated within the real-world setting is consistent with prior phase III clinical trial data, and illustrates the real-world effectiveness of crizotinib.
\end{abstract}

Keywords: crizotinib; real-world utilization; patient outcomes; advanced NSCLC; ALK-positive

\section{Introduction}

Biomarker driven therapies have revolutionized the treatment of non-small cell lung cancer (NSCLC). A genetic alteration of the anaplastic lymphoma kinase (ALK) gene is present in $3-5 \%$ of NSCLCs [1-8]. The first FDA-approved ALK inhibitor for ALK-positive metastatic NSCLC treatment in 2011, crizotinib, has shown significant improvement in progression-free survival (PFS) and tumor responses in patients with metastatic NSCLC who carry the ALK gene rearrangement [9]. Crizotinib is 
an oral ATP competitive selective inhibitor of the ALK, MET, and ROS-1 tyrosine kinases that inhibit tyrosine phosphorylation of activated ALK at nanomolar concentrations $[10,11]$. While crizotinib has demonstrated significant improvement in PFS in phase III studies $[12,13]$, there are less data on the utilization and patient outcomes associated with crizotinib in real-world clinical practice. Our study sought to examine treatment patterns and outcomes of crizotinib utilization in NSCLC patients from U.S. community oncology practices, and to explore the impact of line of therapy and presence of brain metastases on outcomes.

\section{Methods}

Institutional review board approval was received for this retrospective study of patients diagnosed with metastatic NSCLC and treated with crizotinib in The U.S. Oncology Network (USON) of community-based oncology practices, utilizing the iKnowMed ${ }^{\mathrm{TM}}$ (iKM) electronic health record (EHR). iKM is an integrated web-based database and oncology-specific EHR system maintained by McKesson Specialty Health (MSH), that captures outpatient practice encounter histories from approximately 1500 community-based oncology providers across practices in 19 states. The patient identification period consisted of patients initiating crizotinib treatment between 1 September 2011 through 31 October 2014, with follow-up data collected through 31 December 2015. The study time period was selected to capture crizotinib-treated patients post FDA approval, and to ensure sufficient follow-up, including survival outcomes. This study reflects the time during which crizotinib was the only approved first-line agent for ALK-positive patients.

Data for this study was obtained from the iKM database, supplemented with electronic medical chart abstraction, claims data, and vital status from Social Security Death Index (SSDI). Records, including diagnosis date, age, sex, race, practice region, histology, sites of metastases, performance status, tumor markers, comorbidities, and smoking history, were reviewed. Treatment records, including crizotinib doses, treatment dates, and line of therapy were identified.

Duration of therapy, healthcare resource use, and clinical outcomes, including overall survival (OS), and time to treatment failure (TTF), were assessed. OS was defined as the time from initiation of crizotinib until death from any cause. TTF was defined as time from initiation of crizotinib until discontinuation of treatment for any reason (e.g., progression, toxicity, other).

Pre-crizotinib treatment was identified as those regimens given within 30 days prior to the first crizotinib prescription date. Post-crizotinib treatment was identified as those regimens given within 30 days after the last crizotinib prescription date. First line $(1 \mathrm{~L})$ crizotinib treatment was defined as the first identified treatment with crizotinib not preceded by any other treatment for NSCLC therapy. Second line or later $(\geq 2 \mathrm{~L})$ crizotinib treatment was defined as treatment with crizotinib that was preceded by one or more systemic chemotherapeutic regimens for NSCLC.

Healthcare resource utilization that occurred during crizotinib treatment (crizotinib initiation through 30 days after the last identified treatment of crizotinib therapy) was captured utilizing CPT codes, and summarized for the specific categories of healthcare usage (e.g., outpatient visits, laboratory procedures, radiotherapy, imaging).

The cost from claims of each healthcare use was estimated based on the amount allowed to the rendering service provider on the payer remittance per the 2014 Medicare data. These amounts are normalized costs for the treatment of these patient subgroups treated with the MSH/USON community oncology practices. Healthcare expenditure was reported by health resource category for patients overall, and further calculated as per patient per month cost.

Patients were included if they were $\geq 18$ years at initial NSCLC diagnosis, had evidence of metastatic disease, initiated treatment with crizotinib, and received care at an USON practice with full iKM EHR capabilities over the study period. Patients with a concomitant diagnosis of other primary cancers during the study period or enrolled in clinical trials prior to crizotinib initiation were excluded. 


\section{Statistical Methods}

Patient demographic, clinical, and treatment characteristics were summarized. The association between patient characteristics and line of therapy (LOT), presence or absence of brain metastases, were compared with Fisher's exact test (for the categorical variables) and Kruskal-Wallis test (for the continuous variables), as applicable. Survival and time to treatment failure were calculated from the date of initiation (prescription date) of crizotinib with the Kaplan-Meier (KM) method. $p$-Values comparing curves were calculated with log-rank tests.

\section{Results}

\subsection{Demographics and Baseline Characteristics}

A total of 70,300 patients with NSCLC were identified during the study period; 274 metastatic patients initiated treatment with crizotinib during the study period, and 212 met all eligibility criteria. Of those, 199 had a confirmed documented ALK-positive statu.

The majority of physician practices were located in the south (55.8\%) followed by the west $(27.1 \%$; Table 1). The median age at crizotinib initiation was 60.2 years (min-max 27.1-88.2); $54.8 \%$ were never smokers, 33.7\% were former smokers, and 77.4\% had an Eastern Cooperative Oncology Group (ECOG) performance status score of 0 or 1 . Sites of distant metastases were mostly bone (45.7\%) followed by distant lymph nodes $(36.7 \%)$, brain $(32.2 \%)$, and liver $(24.6 \%)$. The majority of patients $(88.9 \%)$ had confirmed adenocarcinoma histology at initial NSCLC diagnosis.

Table 1. Demographics and baseline characteristics.

\begin{tabular}{|c|c|}
\hline Characteristic & All Patients $(n=199)$ \\
\hline Females & $104(52.3)$ \\
\hline \multicolumn{2}{|l|}{ Age at crizotinib initiation, years } \\
\hline Median (min, max) & $60.2(27.1-88.2)$ \\
\hline $18-35$ & $8(4.0)$ \\
\hline $36-45$ & $26(13.1)$ \\
\hline $46-55$ & $38(19.1)$ \\
\hline $56-65$ & $59(29.7)$ \\
\hline$>65$ & $68(34.2)$ \\
\hline \multicolumn{2}{|l|}{ BMI, $\mathrm{kg} / \mathrm{m}^{2}$} \\
\hline Median (min, $\max )$ & $25.6(15.9-50.3)$ \\
\hline \multicolumn{2}{|l|}{ Census regions (physicians), $n(\%)$} \\
\hline South & $111(55.8)$ \\
\hline West & $54(27.1)$ \\
\hline Midwest & $24(12.1)$ \\
\hline Northeast & $10(5.0)$ \\
\hline \multicolumn{2}{|c|}{ ECOG PS at advanced NSCLC diagnosis } \\
\hline 0 & $22(11.1)$ \\
\hline 1 & $132(66.3)$ \\
\hline 2 & $27(13.6)$ \\
\hline 3 & $1(0.5)$ \\
\hline Unknown & $17(8.5)$ \\
\hline \multicolumn{2}{|l|}{ Stage at initial NSCLC diagnosis } \\
\hline Early (stage IA, IB, IIA, IIB) & $16(8.0)$ \\
\hline Limited/regional (stage IIIA) & $15(7.5)$ \\
\hline Locally advanced (stage IIIB) & $22(11.1)$ \\
\hline Metastatic (stage IV) & $133(66.8)$ \\
\hline Missing/unknown & $13(6.5)$ \\
\hline
\end{tabular}


Table 1. Cont.

\begin{tabular}{lc}
\multicolumn{1}{c}{ Characteristic } & All Patients $(\boldsymbol{n}=\mathbf{1 9 9})$ \\
\hline Histology & $177(88.9)$ \\
Adenocarcinoma (mixed or not) & $4(2.0)$ \\
Squamous & $1(0.50)$ \\
Not otherwise specified (NOS) & $17(8.5)$ \\
Missing/unknown & \\
\hline Smokingstatus & $23(11.6)$ \\
Current & $67(33.7)$ \\
Former & $109(54.8)$ \\
Never & \\
\hline Sites of metastases & $18(9.1)$ \\
Adrenal gland & $91(45.7)$ \\
Bone & $64(32.2)$ \\
Brain & $73(36.7)$ \\
Distant lymph nodes & $49(24.6)$ \\
Liver & $120(60.3)$ \\
Other ${ }^{1}$ & \\
\hline 1 Other consists of identification of categories not previously specified (e.g. pleural cavity).
\end{tabular}

\subsection{Crizotinib Treatment Patterns}

Crizotinib was prescribed as $1 \mathrm{~L}$ treatment in $61.8 \%(n=123)$ and $\geq 2 \mathrm{~L}$ in $38.2 \%(n=76)$ of patients (Table 2). Total average duration of crizotinib treatment was 11.5 months (SD =10.6). Treatment of $250 \mathrm{mg}$, twice daily, was most commonly prescribed (89.5\%) at initial treatment in the overall population, and was consistent by LOT and presence or absence of brain metastases. Dose remained unchanged from the initial dose in $79.4 \%$ of all NSCLC patients evaluated.

Table 2. Demographics, baseline characteristics and treatment patterns by crizotinib line of therapy and presence/absence of central nervous system (CNS) metastases.

\begin{tabular}{|c|c|c|c|c|c|c|}
\hline & \multicolumn{3}{|c|}{ Line of Therapy for Crizotinib Initiation } & \multicolumn{3}{|c|}{ CNS Metastases } \\
\hline & $\begin{array}{l}\text { First-Line } \\
(n=123)\end{array}$ & $\begin{array}{l}\text { Second/Later-Line } \\
\quad(n=76)\end{array}$ & $p$-Value & $\begin{array}{l}\text { Present } \\
(n=64)\end{array}$ & $\begin{array}{c}\text { Absent } \\
(n=135)\end{array}$ & $p$-Value \\
\hline \multicolumn{7}{|l|}{ Sex, $n(\%)$} \\
\hline Female & $63(51.2)$ & $41(54.0)$ & 0.77 & $34(53.1)$ & $70(51.9)$ & 0.88 \\
\hline \multicolumn{7}{|l|}{$\begin{array}{l}\text { Age (years) at crizotinib } \\
\text { initiation }\end{array}$} \\
\hline Median (min, max) & $\begin{array}{c}59.3 \\
(27.1,88.2)\end{array}$ & $\begin{array}{c}63.2 \\
(28.3,86.7)\end{array}$ & & $\begin{array}{c}59.4 \\
(28.381 .3)\end{array}$ & $\begin{array}{c}61.5 \\
(27.1,88.2)\end{array}$ & \\
\hline \multicolumn{7}{|l|}{ Age distribution (years) } \\
\hline $18-35$ & $3(2.4)$ & $5(6.6)$ & & $3(4.7)$ & $5(3.7)$ & \\
\hline $36-45$ & 19 (15.5) & $7(9.2)$ & & 11 (17.2) & 15 (11.1) & \\
\hline $46-55$ & 29 (23.6) & $9(11.8)$ & 0.08 & $12(18.8)$ & $26(19.3)$ & 0.47 \\
\hline $56-65$ & $33(26.8)$ & $26(34.2)$ & & 19 (29.7) & 40 (29.6) & \\
\hline$>65$ & $39(31.7)$ & $29(38.2)$ & & $19(29.7)$ & $49(36.3)$ & \\
\hline \multicolumn{7}{|l|}{$\begin{array}{l}\text { Census regions } \\
\text { (physicians), } n \text { (\%) }\end{array}$} \\
\hline Midwest & $17(13.8)$ & $7(9.2)$ & & $8(12.5)$ & $16(11.9)$ & \\
\hline Northeast & $4(3.3)$ & $6(7.9)$ & & $2(3.1)$ & $8(5.9)$ & \\
\hline South & $69(56.1)$ & $42(55.3)$ & 0.42 & $33(51.6)$ & $78(57.8)$ & 0.58 \\
\hline West & $33(26.8)$ & $21(27.6)$ & & $21(32.8)$ & $33(24.4)$ & \\
\hline \multicolumn{7}{|l|}{ BMI } \\
\hline Median (min, max) & $\begin{array}{c}25.7 \\
(15.9,50.3)\end{array}$ & $\begin{array}{c}24.9 \\
(17.3,49.7)\end{array}$ & & $\begin{array}{c}25.8 \\
(17.3,50.3)\end{array}$ & $\begin{array}{c}25.2 \\
(15.9,49.7)\end{array}$ & \\
\hline
\end{tabular}


Table 2. Cont.

\begin{tabular}{|c|c|c|c|c|c|c|c|}
\hline & & \multicolumn{3}{|c|}{ Line of Therapy for Crizotinib Initiation } & \multicolumn{3}{|c|}{ CNS Metastases } \\
\hline & & $\begin{array}{l}\text { First-Line } \\
(n=123)\end{array}$ & $\begin{array}{l}\text { Second/Later-Line } \\
\quad(n=76)\end{array}$ & $p$-Value & $\begin{array}{l}\text { Present } \\
(n=64)\end{array}$ & $\begin{array}{c}\text { Absent } \\
(n=135)\end{array}$ & $p$-Value \\
\hline \multicolumn{8}{|l|}{$\begin{array}{l}\text { ECOG at crizotinib } \\
\text { initiation, } n(\%)\end{array}$} \\
\hline 0 & & $17(13.8)$ & $5(6.6)$ & \multirow{5}{*}{0.23} & $6(9.4)$ & $16(11.9)$ & \multirow{5}{*}{0.23} \\
\hline 1 & & $76(61.8)$ & $56(73.7)$ & & $39(60.9)$ & $93(68.9)$ & \\
\hline 2 & & $18(14.6)$ & $9(11.8)$ & & $13(20.3)$ & 14 (10.4) & \\
\hline 3 & & $1(0.8)$ & 0 & & $0(0.0)$ & $1(0.7)$ & \\
\hline Unknown & & $11(8.9)$ & $6(7.9)$ & & $6(9.4)$ & $11(8.2)$ & \\
\hline \multicolumn{8}{|l|}{ Disease stage at initial } \\
\hline NSCLC diagnosis, $n(\%)$ & & & & & & & \multirow{6}{*}{$<0.01$} \\
\hline $\begin{array}{l}\text { Early (stage IA, IB, IIA, } \\
\text { IIB) }\end{array}$ & & $7(5.7)$ & $9(11.8)$ & \multirow{5}{*}{$<0.01$} & $2(3.1)$ & $14(10.4)$ & \\
\hline $\begin{array}{l}\text { Limited/regional (stage } \\
\text { IIIA) }\end{array}$ & & $6(4.9)$ & $9(11.8)$ & & $6(9.4)$ & $9(6.7)$ & \\
\hline $\begin{array}{l}\text { Locally advanced (stage } \\
\text { IIIB) }\end{array}$ & & $8(6.5)$ & $14(18.4)$ & & $2(3.1)$ & $20(14.8)$ & \\
\hline Metastatic (stage IV) & & $94(76.4)$ & $39(51.3)$ & & $52(81.3)$ & $81(60.0)$ & \\
\hline Unknown & & $8(6.5)$ & $5(6.6)$ & & $2(3.1)$ & $11(8.2)$ & \\
\hline \multicolumn{8}{|l|}{$\begin{array}{l}\text { Histology at crizotinib } \\
\text { initiation }(\%)\end{array}$} \\
\hline $\begin{array}{l}\text { Adenocarcinoma (mixed } \\
\text { or not) }\end{array}$ & & $113(91.9)$ & $64(84.2)$ & \multirow{4}{*}{0.42} & $57(89.1)$ & $120(88.9)$ & \multirow{4}{*}{0.11} \\
\hline Squamous & & $2(1.6)$ & $2(2.6)$ & & $3(4.7)$ & $1(0.7)$ & \\
\hline $\begin{array}{l}\text { Not otherwise specified } \\
\text { (NOS) }\end{array}$ & & $0(0.0)$ & $1(1.3)$ & & $0(0.0)$ & $1(0.7)$ & \\
\hline Missing/Unknown & & $8(6.5)$ & $9(11.8)$ & & $4(6.3)$ & $13(9.6)$ & \\
\hline \multicolumn{8}{|l|}{$\begin{array}{l}\text { Smokingstatus (closest to } \\
\text { crizotinib initiation), } n(\%)\end{array}$} \\
\hline Current smoker & & $11(8.9)$ & $12(15.8)$ & \multirow{3}{*}{0.30} & $7(10.9)$ & $16(11.9)$ & \multirow{3}{*}{0.31} \\
\hline Former smoker & & 41 (33.3) & $26(34.2)$ & & $17(26.6)$ & $50(37.0)$ & \\
\hline Never smoked & & $71(57.7)$ & $38(50.0)$ & & $40(62.5)$ & $69(51.1)$ & \\
\hline \multicolumn{8}{|l|}{$\begin{array}{l}\text { Site(s) of distant metastases } \\
\text { at crizotinib initiation, } n(\%)\end{array}$} \\
\hline Adrenal gland & & $11(8.9)$ & $7(9.2)$ & 1.00 & $6(9.4)$ & $12(8.9)$ & 1.00 \\
\hline Bone & & $56(45.5)$ & $35(46.1)$ & 1.00 & $31(48.4)$ & $60(44.4)$ & 0.65 \\
\hline Brain & & $43(35.0)$ & $21(27.6)$ & 0.35 & $64(100.0)$ & $0(0.0)$ & $<0.01$ \\
\hline Distant lymph nodes & & $46(37.4)$ & $27(35.5)$ & 0.88 & $16(25.0)$ & $57(42.2)$ & 0.02 \\
\hline Liver & & $34(27.6)$ & 15 (19.7) & 0.24 & $18(28.1)$ & $31(23.0)$ & 0.48 \\
\hline Other & & $75(61.0)$ & $45(59.2)$ & 0.88 & $35(54.7)$ & $85(63.0)$ & 0.28 \\
\hline \multicolumn{8}{|l|}{ Treatment Patterns } \\
\hline & \multirow{2}{*}{$\begin{array}{l}\text { Overall } \\
(n=199)\end{array}$} & \multicolumn{2}{|c|}{$\begin{array}{l}\text { Line of Therapy for } \\
\text { Crizotinib Initiation }\end{array}$} & \multirow[t]{2}{*}{$p$-Value } & \multicolumn{3}{|c|}{ CNS Metastases } \\
\hline & & $\begin{array}{l}\text { First-Line } \\
(n=123)\end{array}$ & $\begin{array}{l}\text { Second/Later-Line } \\
\quad(n=76)\end{array}$ & & $\begin{array}{l}\text { Present } \\
(n=64)\end{array}$ & $\begin{array}{c}\text { Absent } \\
(n=135)\end{array}$ & $p$-Value \\
\hline \multicolumn{8}{|l|}{$\begin{array}{l}\text { Total duration of crizotinib } \\
\text { treatment }\end{array}$} \\
\hline Mean (SD), months & $\begin{array}{c}11.5 \\
(10.6)\end{array}$ & $11.0(9.9)$ & $12.3(11.6)$ & \multirow[t]{2}{*}{0.66} & $11.4(9.4)$ & $11.5(11.1)$ & 0.64 \\
\hline Median (range), months & $\begin{array}{c}8.5 \\
(0.2-48.3)\end{array}$ & $\begin{array}{c}8.5 \\
(0.2-46.6)\end{array}$ & $8.4(0.3-48.3)$ & & $\begin{array}{c}10.5 \\
(0.2-33.2)\end{array}$ & $\begin{array}{c}7.9 \\
(0.3-48.3)\end{array}$ & \\
\hline $\begin{array}{l}\text { Total duration of crizotinib } \\
\text { treatment, } n(\%)\end{array}$ & & & & & & & \\
\hline$<3$ months & 49 (24.6) & $30(24.4)$ & $19(25.0)$ & 1.00 & $16(25.0)$ & $33(24.4)$ & 1.00 \\
\hline$\geq 3$ months & $\begin{array}{c}150 \\
(75.4)\end{array}$ & $93(75.6)$ & $57(75.0)$ & 1.00 & $48(75.0)$ & $102(75.6)$ & 1.00 \\
\hline $\begin{array}{l}\text { Cancer treatment received } \\
\text { within } 30 \text { days before } \\
\text { crizotinib start date, } n(\%)\end{array}$ & $52(26.1)$ & $26(21.1)$ & $26(34.2)$ & & $13(20.3)$ & $39(28.9)$ & \\
\hline Platinum doublet ${ }^{1, \pm}$ & $17(32.7)$ & $13(50.0)$ & $4(15.4)$ & & $5(38.5)$ & $12(30.8)$ & \\
\hline Platinum triplet ${ }^{2, \pm}$ & $13(25.0)$ & $7(26.9)$ & $6(23.1)$ & 0.03 & $7(53.8)$ & $6(15.4)$ & 0.94 \\
\hline Pemetrexed ${ }^{ \pm}$ & $8(15.4)$ & $1(3.8)$ & $7(26.9)$ & & $3(23.1)$ & $5(12.8)$ & \\
\hline Erlotinib $^{ \pm}$ & $3(5.8)$ & $1(3.8)$ & $2(7.7)$ & & $0(0.0)$ & $3(7.7)$ & \\
\hline Bevacizumab $^{ \pm}$ & $2(3.9)$ & $0(0.0)$ & $2(7.7)$ & & $0(0.0)$ & $2(5.1)$ & \\
\hline Other ${ }^{3, \pm}$ & $9(17.3)$ & $4(15.4)$ & $5(19.2)$ & & $2(15.4)$ & $7(17.9)$ & \\
\hline
\end{tabular}


Table 2. Cont.

\begin{tabular}{|c|c|c|c|c|c|c|c|}
\hline $\begin{array}{l}\text { Cancer treatment received } \\
\text { within } 30 \text { days post } \\
\text { crizotinib end date, } n(\%)\end{array}$ & $71(35.7)$ & $50(40.7)$ & $21(27.6)$ & 0.21 & $24(37.5)$ & $47(34.8)$ & 0.99 \\
\hline Platinum doublet ${ }^{4, \pm}$ & $16(22.5)$ & $12(24.0)$ & $4(19.0)$ & & $6(25.0)$ & $10(21.3)$ & \\
\hline Platinum triplet $5, \pm$ & $2(2.8)$ & $2(4.0)$ & $0(0.0)$ & & $0(0.0)$ & $2(4.3)$ & \\
\hline Ceritinib $^{ \pm}$ & 29 (40.9) & $22(44.0)$ & $7(33.3)$ & & $10(41.7)$ & $19(40.4)$ & \\
\hline Pemetrexed ${ }^{ \pm}$ & $5(7.0)$ & $2(4.0)$ & $3(14.3)$ & & $2(8.3)$ & $3(6.4)$ & \\
\hline Alectinib $^{ \pm}$ & $2(2.8)$ & $2(4.0)$ & $0(0.0)$ & & $0(0.0)$ & $2(4.3)$ & \\
\hline Docetaxel $^{ \pm}$ & $2(2.8)$ & $0(0.0)$ & $2(9.5)$ & & $1(4.2)$ & $1(2.1)$ & \\
\hline Other ${ }^{6, \pm}$ & $15(21.1)$ & $10(20.0)$ & $5(23.8)$ & & $5(20.8)$ & $10(21.3)$ & \\
\hline \multicolumn{8}{|l|}{$\begin{array}{l}\text { Initial crizotinib total daily } \\
\text { dose, } n(\%)\end{array}$} \\
\hline $250 \mathrm{mg}$ QD & $11(5.5)$ & $5(4.1)$ & $6(7.9)$ & \multirow{3}{*}{0.04} & $3(4.7)$ & $8(5.9)$ & \multirow{3}{*}{1.0} \\
\hline $200 \mathrm{mg}$ BID & $10(5.0)$ & $3(2.4)$ & $7(9.21)$ & & $3(4.7)$ & $7(5.2)$ & \\
\hline $250 \mathrm{mg}$ BID & $\begin{array}{c}178 \\
(89.5)\end{array}$ & $115(93.5)$ & $63(82.9)$ & & $58(90.6)$ & $120(88.9)$ & \\
\hline \multicolumn{8}{|l|}{$\begin{array}{l}\text { Crizotinib total daily dose } \\
\text { changes, } n(\%)\end{array}$} \\
\hline$\geq 1$ dose escalation & $3(1.5)$ & $1(0.8)$ & $2(2.6)$ & \multirow{3}{*}{0.77} & $0(0.0)$ & $3(2.2)$ & \multirow{4}{*}{0.67} \\
\hline$\geq 1$ dose reduction & $26(13.1)$ & $16(13.0)$ & $10(13.2)$ & & $7(10.9)$ & $19(14.1)$ & \\
\hline $\begin{array}{l}\geq 1 \text { dose reduction and } \\
\geq 1 \text { dose escalation }\end{array}$ & $12(6.0)$ & $7(5.7)$ & $5(6.6)$ & & $3(4.7)$ & $9(6.7)$ & \\
\hline No changes & $\begin{array}{c}158 \\
(79.4)\end{array}$ & $99(80.5)$ & $59(77.6)$ & & $54(84.4)$ & $104(77.0)$ & \\
\hline \multicolumn{8}{|l|}{$\begin{array}{l}\text { Other cancer treatment } \\
\text { during active crizotinib } \\
\text { treatment, } n(\%)\end{array}$} \\
\hline Radiotherapy & 37 (18.6) & $24(19.5)$ & $13(17.1)$ & 0.71 & $23(35.9)$ & $14(10.4)$ & \multirow{2}{*}{$\begin{array}{c}<0.01 \\
0.03\end{array}$} \\
\hline Other & $49(24.6)$ & $33(26.8)$ & $16(21.1)$ & 0.40 & $22(34.4)$ & $27(20.0)$ & \\
\hline \multicolumn{8}{|l|}{$\begin{array}{l}\text { Primary reason(s) for final } \\
\text { d/c of crizotinib } 7, n(\%)\end{array}$} \\
\hline Death & $26(16.8)$ & $16(16.7)$ & $10(17.0)$ & \multirow{8}{*}{0.79} & $9(15.8)$ & $17(17.4)$ & \multirow{8}{*}{0.86} \\
\hline Disease progression & $91(58.7)$ & $60(62.5)$ & $31(52.5)$ & & $33(57.9)$ & $58(59.2)$ & \\
\hline $\begin{array}{l}\text { Treatment-related } \\
\text { toxicity or side effects }\end{array}$ & $5(3.2)$ & $2(2.1)$ & $3(5.1)$ & & $1(1.8)$ & $4(4.1)$ & \\
\hline Physician preference & $6(3.9)$ & $3(3.1)$ & $3(5.1)$ & & $3(5.3)$ & $3(3.1)$ & \\
\hline Patient preference & $8(5.2)$ & $4(4.2)$ & $4(6.8)$ & & $3(5.3)$ & $5(5.1)$ & \\
\hline Cost & $1(0.7)$ & $1(1.0)$ & $0(0.0)$ & & $1(1.8)$ & $0(0.0)$ & \\
\hline Other reason & $10(6.5)$ & $6(6.3)$ & $4(6.8)$ & & $3(5.3)$ & $7(7.1)$ & \\
\hline Unknown/missing & $8(5.2)$ & $4(4.2)$ & $4(6.8)$ & & $4(7.0)$ & $4(4.1)$ & \\
\hline \multicolumn{8}{|c|}{$\begin{array}{l}{ }^{1} \text { Patients received carboplatin + pemetrexed }(n=8) \text {, carboplatin }+ \text { paclitaxel }(n=6) \text {, cisplatin }+ \text { pemetrexed }(n=3) ;{ }^{2} \\
\text { Patients received bevacizumab }+ \text { carboplatin }+ \text { paclitaxel }(n=10) \text {, bevacizumab }+ \text { carboplatin }+ \text { pemetrexed }(n=3) ; \\
{ }^{3} \text { Other regimens were any treatments that were not predefined selections during chart review; }{ }^{4} \text { Patients received } \\
\text { carboplatin + pemetrexed }(n=11) \text {, carboplatin }+ \text { paclitaxel }(n=3) \text {, cisplatin }+ \text { pemetrexed }(n=2) ;{ }^{5} \text { Patients received } \\
\text { bevacizumab + carboplatin }+ \text { paclitaxel }(n=2) ;{ }^{6} \text { Other regimens were any treatments that were not predefined } \\
\text { selections during chart review or patients involved in clinical trials } ;{ }^{7} \text { Percentages for reasons of discontinuation of } \\
\text { crizotinib therapy are out of the number of patients with a known end date. Patients without a known end date } \\
\text { were considered to be in an ongoing therapy. }{ }^{+} \text {The focus of the study was to assess crizotinib treatment by } 1 \mathrm{~L} \\
\text { or } \geq 2 \text { L however it was important to capture those treatments occurring prior to and after crizotinib treatment to } \\
\text { better understand the treatment landscape in this population. }{ }^{ \pm} \text {Each percentage is calculated for specific treatment } \\
\text { received out of those patients receiving pre or post crizotinib treatment. }\end{array}$} \\
\hline
\end{tabular}

Dose reductions occurred in $13.1 \%$ of patients. Radiotherapy was given during crizotinib treatment in $18.6 \%$ of patients. Patients with brain metastases were more likely to receive radiotherapy compared to those without brain metastases (35.5\% versus $10.4 \%, p<0.0001)$. The most common treatment received prior to the start of crizotinib was platinum doublet $(32.7 \%)$.

The most common primary discontinuation reason was progression (58.7\%) followed by death $(16.8 \%)$; and $3.2 \%$ of patients were identified as discontinuing crizotinib as a result of treatment-related toxicity. Twenty-two percent of patients in this study were identified as ongoing crizotinib therapy at the end of the study period and $39.2 \%$ of the overall population moved on to a new therapy after crizotinib. Within those patients receiving post crizotinib treatment, the most common treatment received was ceritinib $(40.9 \%)$. 


\subsection{Crizotinib Clinical Outcomes}

Patients were observed for a median of 13.0 months ( $\min -\max =0.03,46.6)$. The proportion of patients with $<3$ months of crizotinib treatment was $24.6 \%$ and $75.4 \%$ for patients with $\geq 3$ months of treatment (Table 2). Median survival in patients treated for at least 3 months was 33.8 months, with 1- and 2-year survival probabilities of 79.0\% (95\% CI: 71.2, 84.9) and 61.3\% (95\% CI: 51.8, 69.4), respectively. Although overall survival time was numerically reduced in patients initiating crizotinib as $2 \mathrm{~L}$ or greater and in patients with CNS lesions present before crizotinib initiation, it was not statistically different ( $p=0.91$ for crizotinib LOT and $p=0.28$ by brain metastases; Figure 1A,B). Median TTF was 10.4 months $(95 \%$ CI: 7.4, 12.2) in the overall population, and was similar by LOT $(p=0.68)$ and brain metastases $(p=0.16$; Figure 2A,B). Median time to treatment failure was 10.4 months $(95 \% \mathrm{CI}=7.3$, 12.3 months) in the patients initiating crizotinib as $1 \mathrm{~L}$ and 8.6 months ( $95 \% \mathrm{CI}=4.5,15.8$ months) for patients initiating crizotinib as $2 \mathrm{~L}$ or later.

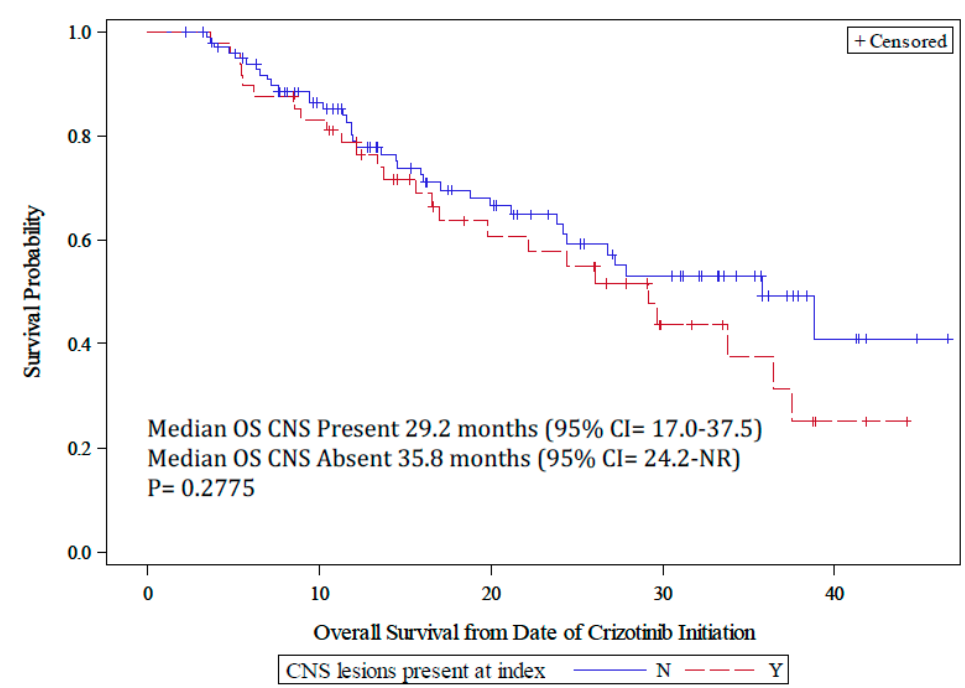

(A)

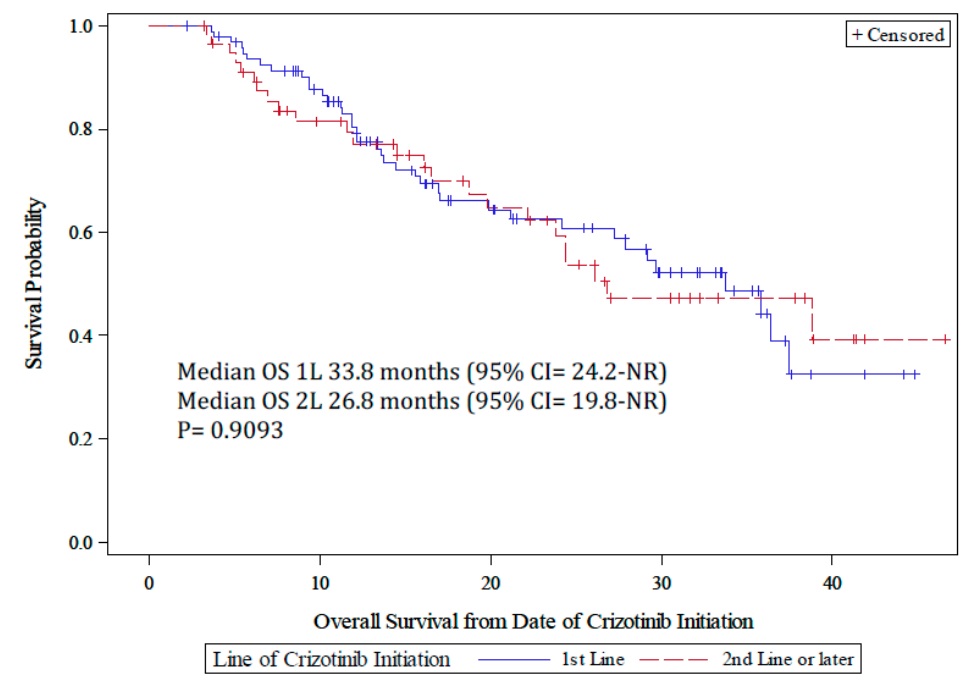

(B)

Figure 1. (A) Kaplan-Meier curve of overall survival from crizotinib initiation by CNS metastases; (B) Kaplan-Meier curve of overall survival from crizotinib initiation by line of therapy (LOT). 


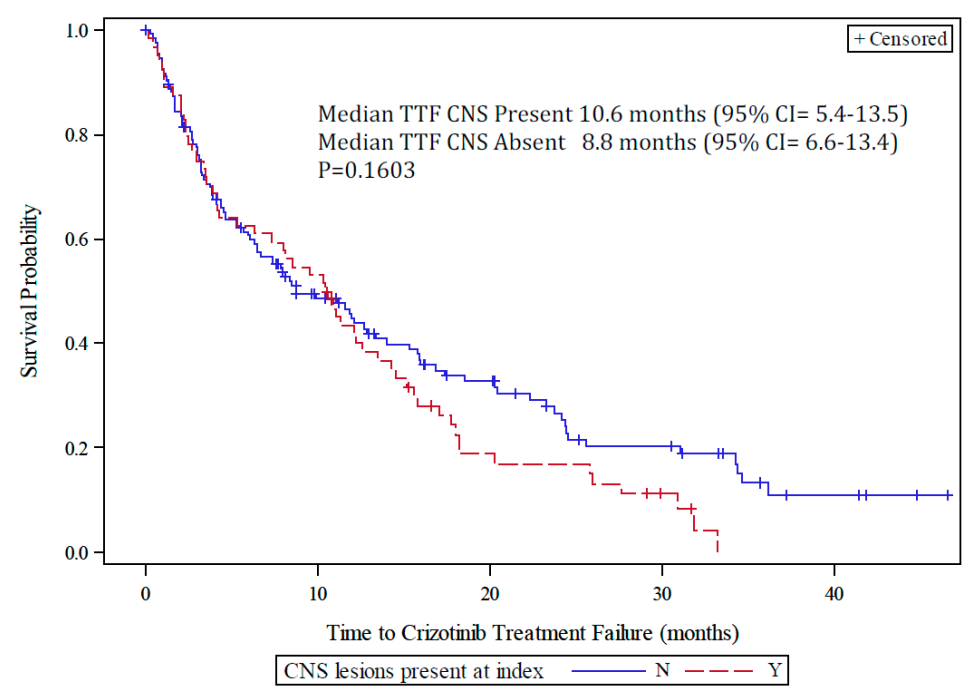

(A)

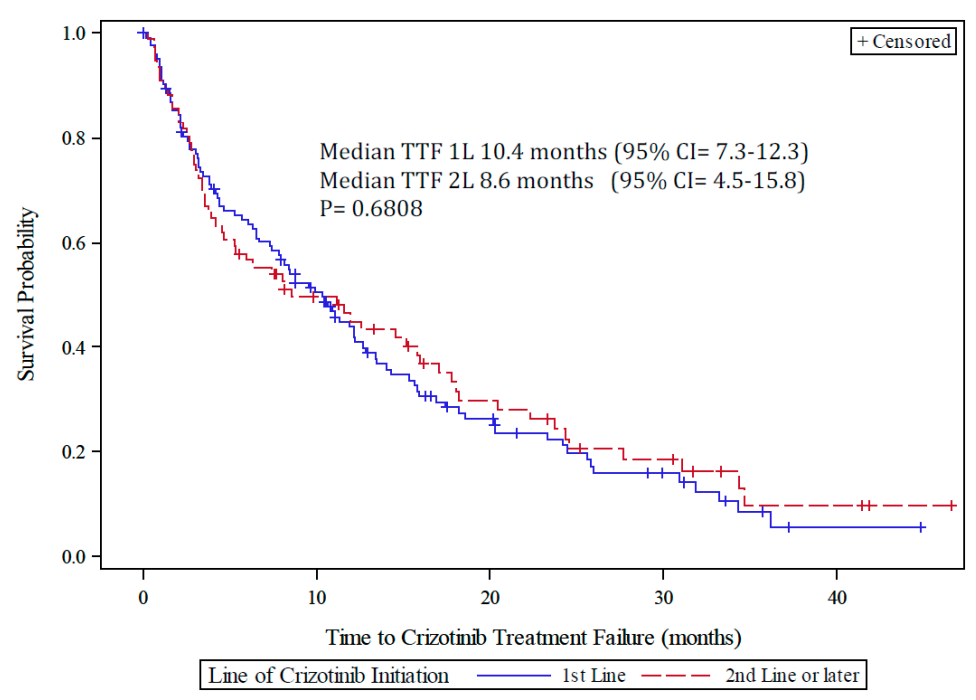

(B)

Figure 2. (A) Kaplan-Meier curve of time to treatment failure from crizotinib initiation by CNS metastases; (B) Kaplan-Meier curve of time to treatment failure from crizotinib initiation by LOT.

\subsection{Healthcare Resource Utilization and Cost}

Claims data during crizotinib treatment was available for 199 patients. Table 3 presents information on healthcare resource use and associated cost overall and by LOT during crizotinib treatment.

Sixty-one $(30.7 \%)$ patients had at least one hospitalization identified from chart review during crizotinib treatment. One hundred fifty-three $(76.9 \%)$ patients had a minimum of one laboratory related claim. Prevalence of at least one claim for radiotherapy and imaging in the overall population during crizotinib treatment consisted of $n=37(18.6 \%)$ and $n=53(26.6 \%)$, respectively. There were no statistically significant differences by crizotinib LOT for categories of healthcare use. 
Table 3. Healthcare resource utilization during crizotinib treatment (reported on a per month basis).

\begin{tabular}{|c|c|c|c|c|}
\hline & \multirow{2}{*}{$\begin{array}{l}\text { Overall } \\
(n=199)\end{array}$} & \multicolumn{3}{|c|}{ Line of Therapy for Crizotinib Initiation } \\
\hline & & $\begin{array}{l}\text { First-Line } \\
(n=123)\end{array}$ & $\begin{array}{l}\text { Second/Later-Line } \\
\quad(n=76)\end{array}$ & $p$-Value \\
\hline \multicolumn{5}{|l|}{$\begin{array}{l}\text { Visits to an emergency room } \\
\text { (on outpatient basis) } 1\end{array}$} \\
\hline $\mathrm{Had} \geq 1$ visit, $n(\%)$ & $28(14.1)$ & $19(15.5)$ & $9(11.8)$ & 0.53 \\
\hline \multicolumn{5}{|l|}{$\begin{array}{l}\text { Hospital admissions } \\
\text { (overnight stay or day } \\
\text { admission excluding ER } \\
\text { visits) for reasons directly } \\
\text { related to NSCLC }{ }^{1}\end{array}$} \\
\hline Had $\geq 1$ admission, $n(\%)$ & $61(30.7)$ & $41(33.3)$ & $20(26.3)$ & 0.34 \\
\hline \multicolumn{5}{|l|}{ Outpatient visits } \\
\hline Had $\geq 1$ claim, $n(\%)$ & $170(85.4)$ & $104(84.6)$ & $66(86.8)$ & 0.84 \\
\hline Median Cost (min, max) & $\begin{array}{c}\$ 108.65 \\
(9.30,336.88)\end{array}$ & $\begin{array}{c}\$ 110.50 \\
(9.30,334.99)\end{array}$ & $\begin{array}{c}\$ 99.38 \\
(18.86,336.88)\end{array}$ & \\
\hline \multicolumn{5}{|l|}{ Laboratory procedures } \\
\hline Had $\geq 1$ claim, $n(\%)$ & $153(76.9)$ & $90(73.2)$ & $63(82.9)$ & 0.12 \\
\hline Median Cost (min, max) & $\begin{array}{c}\$ 26.12 \\
(1.08,153.49)\end{array}$ & $\begin{array}{c}\$ 25.24 \\
(1.08,153.49)\end{array}$ & $\begin{array}{c}\$ 26.29 \\
(2.32,127.76)\end{array}$ & \\
\hline \multicolumn{5}{|l|}{ Radiotherapy } \\
\hline Had $\geq 1$ claim, $n(\%)$ & $37(18.6)$ & $24(19.5)$ & $13(17.1)$ & 0.71 \\
\hline Median Cost (min, max) & $\begin{array}{c}\$ 268.71 \\
(7.63,1613.79)\end{array}$ & $\begin{array}{c}\$ 276.09 \\
(7.63,1613.79)\end{array}$ & $\begin{array}{c}\$ 219.90 \\
(54.65,576.38)\end{array}$ & \\
\hline \multicolumn{5}{|l|}{ Imaging 2} \\
\hline Had $\geq 1$ claim, $n(\%)$ & $53(26.6)$ & $31(25.2)$ & $22(29.0)$ & 0.76 \\
\hline Median Cost (min, max) & $\begin{array}{c}\$ 69.84 \\
(4.43,206.86)\end{array}$ & $\begin{array}{c}\$ 64.17 \\
(4.43,180.72)\end{array}$ & $\begin{array}{c}\$ 71.72 \\
(6.97,206.86)\end{array}$ & \\
\hline
\end{tabular}

${ }^{1}$ Emergency visits and hospitalization costs could not be calculated as inpatient claims data were not captured. Utilization was captured from chart review. ${ }^{2}$ Imaging procedures with a zero cost allowance in the 2014 Medicare data were counted as utilizations but not included in the counts for total cost.

\section{Discussion}

This study examined the real-world treatment patterns and outcomes of patients with metastatic NSCLC treated with crizotinib in a U.S. community oncology setting. Broader real-world effectiveness, safety, and cost data may aid in improving quality and delivery of care, as well as outcomes, by accelerating current understanding of how best to incorporate treatments into everyday clinical practice.

Use of EHR data from a large network of practices allowed for increased sample size of a relatively less common type of NSCLC.

A total of 199 ALK-positive patients receiving crizotinib were included in the study. While the estimated prevalence of ALK-positive NSCLC is approximately 3-5\%, only approximately $10 \%$ of those expected to be ALK-positive were identified and treated during the study period. The study period reflected the time frame immediately following approval of crizotinib as the first ALK-inhibitor, so not all patients may have been tested, or could have been tested for the ALK gene rearrangement, if there was insufficient tissue or if patients were not candidates for re-biopsy. However, it does raise the importance of education and awareness of biomarker testing. As the emergence of the benefit of ALK targeted therapy, and new second generation ALK inhibitors have emerged, we would anticipate this number to increase over time.

Consistent with prior studies [12,13], our population was predominately female (52.3\%), never $(54.8 \%)$ or former smokers $(33.7 \%)$, with a greater prevalence of adenocarcinoma histology $(88.9 \%)$. 
Our population was on average older ( 60 vs $51-52$ years) than what has been reported by other studies [12,13].

The most common reason for treatment discontinuation in this population was disease progression, however, the study by Solomon et al. [13] documented that $73 \%$ of patients with progressive disease continued to receive crizotinib as $1 \mathrm{~L}$ beyond Response Evaluation Criteria in Solid Tumors (RECIST) defined progression.

Additionally, treatment appeared to be well tolerated, as $3.2 \%$ of patients discontinued as a result of treatment-related toxicity. This was similar to the results from Solomon et al. [13], as patients treated with crizotinib in that study tended to have low grade toxic events (grades 1-2) and 12\% of patients permanently discontinued crizotinib treatment resulting from adverse events with varying etiology.

With a median follow-up time of 13.0 months, median OS from crizotinib initiation in patients treated with crizotinib for at least 3 months was 33.8 months with 1- and 2-year survival rates of $79.0 \%$ and $61.3 \%$, and did not differ by presence of brain metastases or LOT. Overall survival was calculated for patients treated with crizotinib for a minimum of 3 months' duration, to adequately assess the effectiveness of crizotinib treatment. Relative to a previously published phase III clinical trial [12], this study had a longer follow-up, providing further evidence of crizotinib treatment real-world effectiveness for metastatic NSCLC. Prior studies evaluating survival identified estimates for OS as 21.7 months in second-line treatment in one study, while median OS was not reached in another first-line phase III study $[13,14]$.

Overall, our population had a survival rate of $79.4 \%$ at 1 year, and $61.6 \%$ at 2 years. These estimates did not differ by the strata of interest, and are consistent with estimates from another phase III study [13].

Sixty-one (30.7\%) patients in the overall population had at least one hospitalization identified during chart review related to NSCLC during crizotinib treatment. A study conducted by Karve et al. [15] assessing health resource utilization in metastatic NSCLC patients, using administrative claims data, found that $44.9 \%$ of NSCLC patients had at least one hospital admission claim related to NSCLC. The major difference between the studies is the current study specifically evaluated hospital admissions related to NSCLC during crizotinib treatment, whereas the study conducted by Karve et al. [15] evaluated admission claims related to NSCLC which encompassed greater variability in treatment patterns.

\section{Study Limitations}

Limitations include the retrospective nature of this study, and potential for documentation bias if there were errors or omissions in the medical record. The iKM system is used for clinical practice reasons, not solely for research purposes, which may impede the standardization of the data collection methods and instruments. Not all community oncology practices are included in the iKM dataset, and not all of the U.S. Oncology clinics utilize iKM. The study population consists of patients solely treated within the USON network, and these patients may not represent the general patient population with metastatic NSCLC outside the network. As crizotinib is an oral medication, adherence and precise treatment start and stop dates may not be recorded in the EHR and duration of treatment is assessed as best captured in the records.

\section{Conclusions}

Crizotinib usage evaluated within the real-world setting is consistent with prior phase III crizotinib clinical trial data illustrating the treatment effectiveness of crizotinib. The lack of deviation from the initial starting dose suggests treatment tolerability.

Author Contributions: J.B.-S., J.L.E., and M.B. contributed to study design, analysis, interpretation of study results and manuscript development. C.R. contributed to study design and interpretation of study results. E.T.M., J.M., and M.C. contributed to study design, interpretation of study results and manuscript development. 
Acknowledgments: The authors would like to acknowledge Ruslan Horblyuk and Jennifer Frytak who contributed to the study concept and design. This study was sponsored by Pfizer Inc.

Conflicts of Interest: J.B.-S., J.L.E., and M.B. are employees of The US Oncology Network/McKesson Specialty Health who were paid consultants to Pfizer in connection with the development of this manuscript. C.R. was an employee of The US Oncology Network at the time the study was conducted. E.T.M., J.M., and M.C. are employees of Pfizer Inc.

\section{References}

1. Soda, M.; Choi, Y.L.; Enomoto, M.; Takada, S.; Yamashita, Y.; Ishikawa, S.; Fujiwara, S.; Watanabe, H.; Kurashina, K.; Hatanaka, H.; et al. Identification of the transforming EML4-ALK fusion gene in non-small-cell lung cancer. Nature 2007, 448, 561-566. [CrossRef] [PubMed]

2. Garber, K. ALK, lung cancer, and personalized therapy: Portent of the future? J. Natl. Cancer Inst. 2010, 102, 672-675. [CrossRef] [PubMed]

3. Takeuchi, K.; Choi, Y.L.; Soda, M.; Inamura, K.; Togashi, Y.; Hatano, S.; Enomoto, M.; Takada, S.; Yamashita, Y.; Satoh, Y.; et al. Multiplex reverse transcription-PCR screening for EML4-ALK fusion transcripts. Clin. Cancer Res. 2008, 14, 6618-6624. [CrossRef] [PubMed]

4. Palmer, R.H.; Vernersson, E.; Grabbe, C.; Hallberg, B. Anaplastic lymphoma kinase: Signaling in development and disease. Biochem. J. 2009, 420, 345-361. [CrossRef] [PubMed]

5. Mosse, Y.P.; Wood, A.; Maris, J.M. Inhibition of ALK signaling for cancer therapy. Clin. Cancer Res. 2009, 15, 5609-5614. [CrossRef] [PubMed]

6. Sasaki, T.; Rodig, S.J.; Chirieac, L.R.; Jänne, P.A. The biology and treatment of EML4-ALK non-small cell lung cancer. Eur. J. Cancer 2010, 46, 1773-1780. [CrossRef] [PubMed]

7. Koivunen, J.P.; Mermel, C.; Zejnullahu, K.; Murphy, C.; Lifshits, E.; Holmes, A.J.; Choi, H.G.; Kim, J.; Chiang, D.; Thomas, R.; et al. EML4-ALK fusion gene and efficacy of an ALK kinase inhibitor in lung cancer. Clin. Cancer Res. 2008, 14, 4275-4283. [CrossRef] [PubMed]

8. Wong, D.W.; Leung, E.L.; So, K.K.; Tam, I.Y.; Sihoe, A.D.; Cheng, L.C.; Ho, K.K.; Au, J.S.; Chung, L.P.; Pik Wong, M. The EML4-ALK fusion gene is involved in various histologic types of lung cancers from nonsmokers with wild-type EGFR and KRAS. Cancer 2009, 115, 1723-1733. [CrossRef] [PubMed]

9. Schein, P.S.; Scheffler, B. Barriers to efficient development of cancer therapies. Clin. Cancer Res. 2006, 12, 3243-3248. [CrossRef] [PubMed]

10. McDermott, U.; Iafrate, A.J.; Gray, N.S.; Shioda, T.; Classon, M.; Maheswaran, S.; Zhou, W.; Choi, H.G.; Smith, S.L.; Dowell, L.; et al. Genomic alterations of anaplastic lymphoma kinase may sensitize tumors to anaplastic lymphoma kinase inhibitors. Cancer Res. 2008, 68, 3389-3395. [CrossRef] [PubMed]

11. Christensen, J.G.; Zou, H.Y.; Arango, M.E.; Li, Q.; Lee, J.H.; McDonnell, S.R.; Yamazaki, S.; Alton, G.R.; Mroczkowski, B.; Los, G. Cytoreductive antitumor activity of PF-2341066, a novel inhibitor of anaplastic lymphoma kinase and c-Met, in experimental models of anaplastic large-cell lymphoma. Mol. Cancer Ther. 2007, 6, 3314-3322. [CrossRef] [PubMed]

12. Shaw, A.T.; Kim, D.W.; Nakagawa, K.; Seto, T.; Crino, L.; Ahn, M.J.; De Pas, T.; Besse, B.; Solomon, B.J.; Blackhall, F.; et al. Crizotinib versus chemotherapy in advanced ALK-positive lung cancer. N. Engl. J. Med. 2013, 368, 2385-2394. [CrossRef] [PubMed]

13. Solomon, B.J.; Mok, T.; Kim, D.W.; Wu, Y.L.; Nakagawa, K.; Mekhail, T.; Felip, E.; Cappuzzo, F.; Paolini, J.; Usari, T.; et al. First-line crizotinib versus chemotherapy in ALK-positive lung cancer. N. Engl. J. Med. 2014, 371, 2167-2177. [CrossRef] [PubMed]

14. Shaw, A.T.; Janne, P.A.; Besse, B.; Solomon, B.J.; Blackhall, F.H. Crizotinib vs. chemotherapy in ALK+ advanced non-small cell lung cancer (NSCLC): Final survival results from PROFILE 1007. J. Clin. Oncol. 2016, 34, 9066.

15. Karve, S.J.; Price, G.L.; Davis, K.L.; Pohl, G.M.; Smyth, M.N.; Bowman, L. Comparison of demographics, treatment patterns, health care utilization, and costs among elderly patients with extensive-stage small cell and metastatic non-small cell lung cancers. BMC Health Serv. Res. 2014, 14, 555. [CrossRef] [PubMed] 